\title{
Multi-Modal Biometric: Bi-Directional Empirical Mode Decomposition with Hilbert-Hung Transformation
}

\author{
Gavisiddappa $^{1}$ \\ Channabasaveshwara Institute of Technology \\ Gubbi, India \\ ORCID 0000-0003-3385-2640 \\ Chandrashekar Mohan Patil ${ }^{2}$ \\ Vidyavardhaka College of Engineering, \\ Mysore, India \\ ORCID 0000-0002-5220-4799
}

\author{
Shivakumar Mahadevappa ${ }^{3}$ \\ GSSS Institute of Engineering and Technology for Women \\ Mysore, India \\ ORCID 0000-0003-1247-6009
}

\author{
Pramod KumarS ${ }^{4}$ \\ Kalpataru Institute of Technology \\ Tiptur, India \\ ORCID 0000-0001-7457-3432
}

\begin{abstract}
Biometric systems (BS) helps in reorganization of individual person based on the biological traits like ears, veins, signatures, voices, typing styles, gaits, etc. As, the Uni-modal BS does not give better security and recognition accuracy, the multimodal BS is introduced. In this paper, biological characters like face, finger print and iris are used in the feature level fusion based multimodal BS to overcome those issues. The feature extraction is performed by Bi-directional Empirical Mode Decomposition (BEMD) and Grey Level Co-occurrence Matrix (GLCM) algorithm. Hilbert-Huang transform (HHT) is applied after feature extraction to obtain local features such as local amplitude and phase. The combination of BEMD, HHT and GLCM are used for achieving effective accuracy in the classification process. MMB-BEMD-HHT method is used in Multiclass support vector machine technique (MC-SVM) as a classifier. The false rejection ratio has improved using feature level fusion (FLF) and MC-SVM technique. The performance of MMBBEMD-HHT method is measured based on the parameters like False Acceptance Ratio (FAR), False Rejection Ratio (FRR), and accuracy and compared it with an existing system. The MMBBEMD-HHT method gave $96 \%$ of accuracy for identifying the biometric traits of individual persons.
\end{abstract}

Keywords-Biometric Systems (BS); multimodal biometrics; bidirectional empirical mode decomposition; Hilbert-Huang transform; Multi-Class Support Vector Machines technique (MC-SVM); 2000 Mathematics Subject Classification: 92C55, 94A08, $92 C 10$

\section{INTRODUCTION}

MMBS uses two or more physiological or behavioural characteristics for identification and it is more convenient than traditional authentication techniques. The main objective of the MMBS is to minimize the False Acceptance Rate (FAR), False Rejection Rate (FRR) and Failure To Enroll Rate (FTE) [1], [2]. In different orientations, the directional bank filter involves to extract the palm vein patterns and also the non-vein pixels are identified by assessing the Directional Filtering Magnitude (DFM) [3]. Feature level fusion (FLF) helps to extract the features from fingerprint and face. Then the impact over the matching performance is analysed using the random projection based transformation and proportion weight factor. Then FLF fuses the off-line signature along with curvelet transform is used for feature extraction [4], [5]. A fused multimodal system like FLF is used over the fingerprint, retina and finger vein to reduce the genuine acceptance rate (GAR) and also the security against spoofing is improved [6]. A chaff point based fuzzy vault extracts the features of face and ear points and the best point is created by selecting the best locations of feature vectors which is found by the particle swarm optimization (PSO) [7]. The fusion over the face and voice modules made by the transformation based fusion algorithm over an ensemble classifier and also the fusion of these behaviour traits are made by Adaptive Neuro Fuzzy Inference System (ANFIS) [8], [9].

The two circular detection detects the iris and then the features of face and iris are combined with support vector machine [10]. Fuzzy vault template is added to enhance the security based on the biometric features of the fingerprint and iris [11]. An adaptive combination of multiple biometric modalities is achieved by using palm print, finger-knuckle print and iris along with PSO is used for optimizing the selection of score level combination, decision threshold and corresponding parameters [12]. Score level fusion occurs over the finger vein, fingerprint, finger shape and finger knuckle print features from the human and it performs based on the triangular norm. Features from the score level fusion may not be compatible and large dimensionality of feature space leads to irrelevant and redundant data [13]. The fuzzy-weighted image quality assessment fuse five types of biometric features such as hand geometry, palm print, palmar knuckle print, palm vein and finger vein. During the fusion, more weights are combined with the images to attain better quality. The vein images are not constant at all times; it changes based on the medical condition [14]. Match score level fusion combines the features of face and signature. Face and signature features are extracted by the Long Term Potention (LTP) and Hidden Markov model (HMM) respectively. Accuracy of the MMBS is not compatible, when the system does not have perfect matching [15]. The existing systems have some limitations like less accuracy, less sensitivity and recall.In order to overcome 
that, the MMB-BEMD-HHT method is developed to enhance the accuracy for identifying the individual biometric traits. The major contributions of this research work is stated as follows:

1) To improve the accuracy of the image recognition, two different feature extraction techniques are combined here such as HHT and GLCM.

2) The FLF is used for fusing the feature vectors of finger print, face and iris. This FLF is pre classification technique which is used before matching and it gives more accurate results when compared to post classification techniques such as score level and decision level fusion. Because the FLF provides the richer information about the extracted feature vectors.

3) Then the classification of features performs by placing the hyperplanes among the margins in MC-SVM.

This research work is composed as follows, Section 2 presents an extensive survey of recent papers based on MMBS. Section 3 briefly described the MM-BS using feature level fusion with MC-SVM based matching. Section 4 describes about an experimental result of a MMB-BEMD-HHT and conventional methods. The conclusion of this research work is given at the end.

\section{LitERATURE SURVEY}

Yang et al. [16] presented the cancellable multi-biometric system that comprises of fingerprint and finger vein and it provided the template protection and revocability. Fingerprint and fingerprint vein were extracted by the alignment of free local structures and image based technique respectively. The minutia based fingerprint feature set and image based finger vein feature set were fused by FLF which have three different kinds of fusion options. This method was used to improve the GAR. The multi-biometric system affects by non-linear distortion and the noise which present in the fingerprint image.

Puneet et al. [17] introduced the multi-modal (MM) authentication which depends on the palm-dorsa vein pattern. The fusion which is present in this MM have four types such as multi-algorithm fusion, data fusion, feature fusion and score fusion. The genuine vein patterns were extracted by multialgorithm fusion and the false vein patterns were cancelled from the extracted vein patterns via fusion. There are three types of features are extracted from the vein pattern such as shape features, minutiae and features of hand boundary shape. Finally, third level of fusion was applied for fusing the minutiae and shape features. These three fusion algorithms were used to improve the accuracy, but this system is unstable because of the elastic nature of the skin.

Xiaojun et al. [18] improved the feature level of MM-BS fusion using a multi-set generalized canonical discriminant projection (MGCDP) method. This MGCDP increased the correlation of intra class features during the minimization of correlation among the class. More than two types of BM features were fused with serial MGCDP (S-MGCDP) and parallel MGCDP (P-MGCDP). This MGCDP was used to improve the recognition rate that has ability to identify the new samples.

Asaari et. al. [19] considered the fusion of finger vein and finger geometry recognition to introduce a multimodal finger biometrics. The Band Limited Phase Only Correlation (BLPOC) determined the similarity between the finger vein images and the recognition of finger geometry using the widthcentroid contour distance (WCCD) combined with centroid contour distance (CCD). The fusion of WCCD and CCD improved the accuracy of finger geometry recognition compared to the single type of feature. If the non-linear distortion is present in finger-vein images, it affects the performance of BLPOC-based finger vein matching [20], [21].

The issue over the non-linear distortion overcome by an ideal high pass filter in MMB-BEMD-HHT method and then the recognition rateis improved by using two different feature extraction techniques such as HHT and GLCM. The MMBBEMD-HHT is clearly explained in Section 3.

\section{MMB-BEMD-HHT METHODOLOGY}

Multi-modal BS are high-performance security systems. This research work introduced a new MMB-BEMD-HHT to improve the classification accuracy. This MMB-BEMD-HHT method involves two major process database creation and testing. Fig. 1 shows the MMB-BEMD-HHT method.

\section{A. MMB-BEMD-HHT Training}

The MMB-BEMD-HHT Training section consists of six major steps namely image acquisition, pre-processing, hybrid feature extraction, fusion and database generation. Three different combinations of inputs such as face, iris, and fingerprint are taken for training. The input images are enhanced with the help of Sharpening filter. The enhanced feature values are extracted by the hybrid feature extraction technique (HHT and GLCM), the feature values, and added by the fusion technique i.e. FLF. Finally, the fused vectors of the images are stored in the database and then these features are tested in MCSVM testing for identifying the features of individuals.

1) Image Acquisition: In this MMB-BEMD-HHT method, three types of biological characters such as face, iris, and fingerprint are taken to identify the individual's biological characters that are presented in Fig. 2. The face and iris images are captured by digital mobile camera and the fingerprint image is captured from the scanner.

2) Pre-processing: A high-pass filter used in this preprocessing for an image enhancement. These filters emphasize fine details in the image and the quality of an image highly degrades when the high frequencies are attenuated or completely removed. The high-frequency components of an image are enhanced that improve the image quality. For example, if the face image is given as an input, then the filter functions for an ideal high pass filter is expressed in Eq. (1):

$$
X_{F}(m, n)=\left\{\begin{array}{ccc}
0 & \text { if } & D(m, n) \leq D 0 \\
1 & \text { if } & D(m, n)>D 0
\end{array}\right.
$$

Where $D(m, n)$ is the distance between the centre of frequency rectangle and $X_{F}(m, n)$ is the enhanced image. Similarly, the iris and fingerprint images are enhanced by this filter and it is expressed as $X_{I}(m, n)$ and $X_{F p}(m, n)$, respectively. 


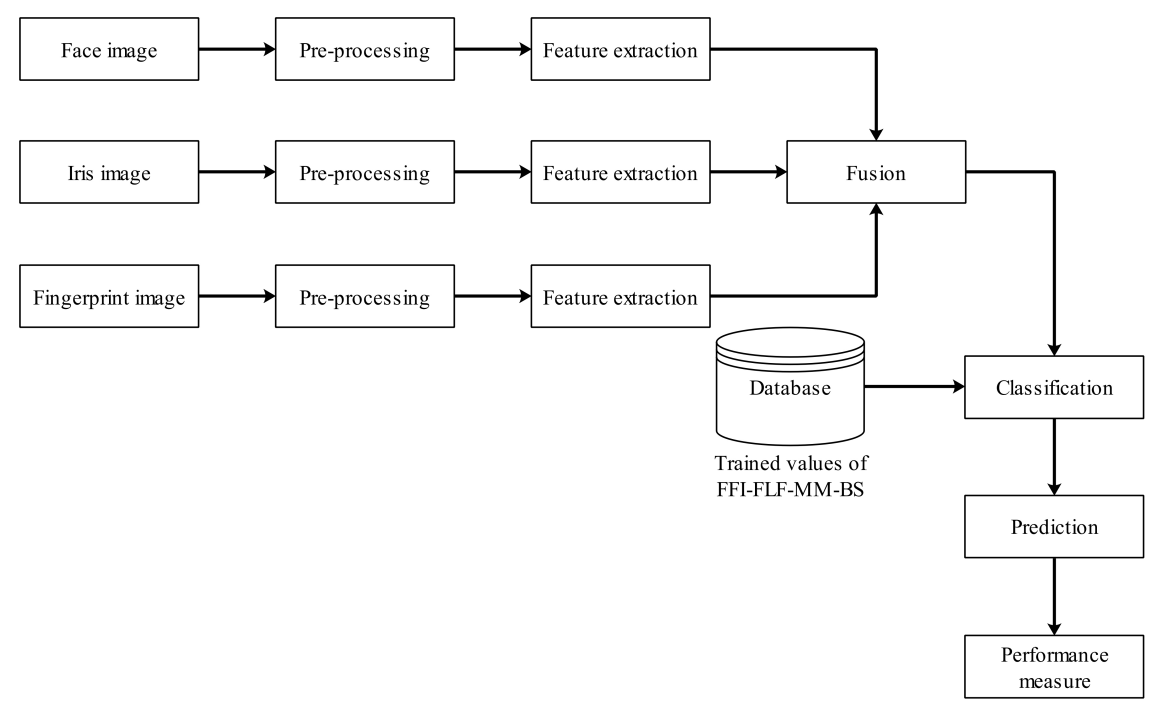

Fig. 1. Block diagram for MMB-BEMD-HHT
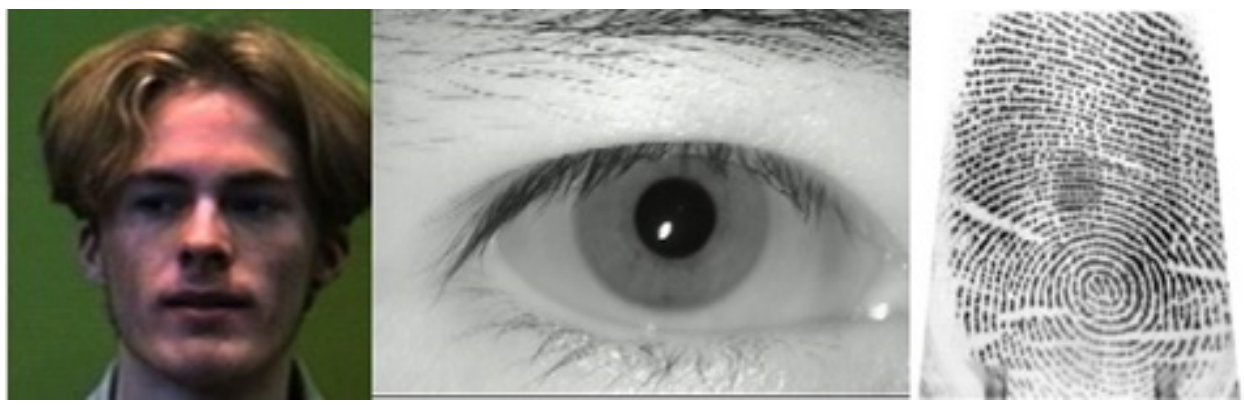

Fig. 2. Image acquisition

3) Hybrid Feature Extraction Technique: The preprocessed images are sent to the hybrid feature extraction. The complicated data set can be decomposed into finite and small number of Intrinsic Mode Function (IMF) components that become the basis representing the data. IMF and residues features of the face, iris and fingerprint are extracted by BEMD and then the Hilbert transform is achieved to handle both non stationary non linear values. Here GLCM also used for extracting the statistical texture features from the preprocessed images. Then the features of GLCM are combined with features from HHT by using FLF.

IMF and residue feature extraction using BEMD: The features of the face, iris and fingerprint images are extracted by BEMD. The nonlinear and non-stationary data are processed by Empirical Mode Decomposition (EMD). The EMD provides sum of oscillatory functions by decomposing the signal and the oscillatory functions are called as Intrinsic Mode Function (IMF). This IMF satisfies the two conditions which are given below:

- The amount of extreme and the amount of zero crossing of an entire data set are either equal or differ at most by one.

- Local minima define the envelope mean value at any point.
From these two conditions, a meaningful instantaneous frequency is calculated. This EMD decomposes a signal $(X(t))$ into a set of IMF's by shifting process. This EMD is also used in the decomposition of images or 2D data which is defined as Bi-dimensional EMD.

An image $X_{F}(m, n)$ is an enhanced face image and the steps of BEMD feature extraction is given as follows:

1) The local minima and maxima of the image $X(m, n)$ are identified.

2) Upper envelope $\left(X_{F u p}(m, n)\right)$ and lower envelope $\left(X_{\text {Flow }}(m, n)\right)$ are obtained by using the cubic spline interpolation between the local minima and maxima.

3) The following Eq. (2) is used to calculate the mean of upper and lower envelope.

$$
S(m, n)=\frac{X_{F u p}(m, n)+X_{F l o w}(m, n)}{2} b
$$

4) The signal $X_{1 F}(m, n)$ is achieved by subtracting the $S(m, n)$ from $X_{F}(m, n)$ and the mathematical expression for $X_{1 F}(m, n)$ is given in Eq. (3).

$$
X_{1 F}(m, n)=X_{F}(m, n)-S(m, n)
$$


5) Then check if $X_{1 F}(m, n)$ accepts the criteria for an IMF. If it is not satisfy means, $X_{F}(m, n)$ is replaced by $X_{1 F}(m, n)$ and repeats the process to achieve the IMF.

The first IMF from the BEMD is in Eq. (4).

$$
C_{1 F}(m, n)=X_{1 F}(m, n)
$$

Where, the intrinsic mode function is denoted as $C_{1 F}(m, n)$. The residue $\left(r_{f}\right)$ is obtained by subtracting the $C_{1 F}(m, n)$ from $X_{F}(m, n)$, which is shown in Eq. (5).

$$
r_{f}(m, n)=X_{F}(m, n)-C_{1 F}(m, n)
$$

Based on the residue value from Eq. (5), the next IMF values are calculated.The shifting process is continued until the final residue becomes constant or it is a function which includes only one maxima or minima from that no more IMF's are obtained. The texture feature of each IMF is denoted by fractal dimensions. Here three IMF's $\left(C_{1 F}, C_{2 F}\right.$ and $\left.C_{3 F}\right)$ and one residue $\left(r_{f}\right)$ value are obtained from an enhanced face image. Similarly, the IMF's and residue from the iris and fingerprint are extracted. The IMF's from the iris and fingerprint are $\left(C_{1 I}, C_{2 I}\right.$ and $\left.C_{3 I}\right)$ and $\left(C_{1 F p}, C_{2 F p}\right.$ and $C_{3 F p}$ ) respectively as well as $r_{i}$ is the residue of iris and $r_{f p}$ is the residue of fingerprint. Then these IMF and residue values of face, iris and fingerprint are given to the input for HHT to extract the local phase and amplitude.

Hilbert-Huang transform: The IMF's and residue from the BEMD are given to HHT for achieving the local features such as amplitude and phase. A 2D analytical signals are obtained by using Riesz transform on the 2D IMF's and residue of the BEMD. Riesz transform are family of generations Hilbert Huang transforms. The generalization of Hilbert transform is the combination of image and its transform is called as monogenic signal. Here, the partial Hilbert transform is performed on the IMF's and residue with respect to a half space which is chosen by introducing a preference direction and it is given in Eq. (6).

For example the first IMF value of Eq. (4) is taken to find the Hilbert transform.

$$
H\left(\overrightarrow{C_{1 F}}\right)=j \operatorname{sign}\left(\overrightarrow{C_{1 F}}, \vec{d}\right)
$$

where, $\vec{d}$ is one preference direction. This Hilbert transform missed the isotropy.

Furthermore, the Riesz transform is utilized in the Hilbert transform and a multidimensional generalization of the Hilbert transform. The frequency domain expression of Riesz transformed signal is in Eq. (7).

$$
F_{R}\left(\overrightarrow{C_{1 F}}\right)=\frac{i \overrightarrow{C_{1 F}}}{\overrightarrow{C_{1 F}}} F\left(\overrightarrow{C_{1 F}}\right)=H\left(\overrightarrow{C_{1 F}}\right) F\left(\overrightarrow{C_{1 F}}\right)
$$

The sparse representation of Eq. (7) is shown in following Eq. (8).

$$
f_{R F 1}(\vec{y})=-\frac{\vec{y}}{2 \pi|\vec{y}|^{3}} \times f_{1 F}(\vec{y})=h(\vec{y}) \times f_{1 F}(\vec{y})
$$

The 2D analytical signal is constituted by Riesz transformed signal and the original signal, and this analytical signal is monogenic signal which is in Eq. (9).

$$
f_{M F 1}(\vec{y})=f_{1 F}(\vec{y})-(i, j) f_{R F 1}(\vec{y})
$$

The above formulation is Eq. (9) 2D analytical signal which is a $3 \mathrm{D}$ vector as well as it should used for achieving local features of monogenic signal.

Phase: The phase of the 2D analytical signal of first IMF is given in Eq. (10).

$$
\operatorname{atan} 3(x, y)=\frac{\vec{y}_{D}}{\left|\vec{y}_{D}\right|} \operatorname{atan}\left(\frac{\left|\vec{y}_{D}\right|}{\left.<(0,0,1)^{T}, \vec{y}\right\rangle}\right)
$$

Where the direction of the rotation vector is $\vec{y}_{D}=(0,0,1)^{T} \times \vec{y}$. The phase of monogenic signal is in Eq. (11).

$$
\varphi_{1 F}(\vec{y})=\operatorname{atan} 3\left(f_{M F 1}(\vec{y})\right)=\arg \left(f_{M F 1}(\vec{y})\right)
$$

Amplitude: The local amplitude of $f_{M F 1}(\vec{y})$ is in Eq. (12)

$$
\begin{array}{r}
\left|f_{M F 1}(\vec{y})\right|=\sqrt{f_{M F 1}(\vec{y}) \overline{f_{M F 1}(\vec{y})}} \\
=\sqrt{f_{1 F}^{2}(\vec{y})+\left|f_{R F 1}(\vec{y})\right|^{2}}
\end{array}
$$

The following Eq. (14) is reconstructed from Eq. (12).

$$
\left.f_{M F 1}(\vec{y})=\left|f_{M F 1}(\vec{y})\right| \exp (-j, i, 0) \varphi_{1 F}(y)\right)
$$

The monogenic signal of each IMF allows to calculate local amplitude and local phase. Likewise, the local phase and amplitude is calculated for remaining two IMF and residue values which is denoted as $\varphi_{1 F}, \varphi_{2 F}, \varphi_{3 F}, \varphi_{4 F}$ and $f_{M F 1,} f_{M F 2}, f_{M F 3}, f_{M F 4}$, respectively. These local phase and amplitude values are combined in one array which is shown in the following Eq. (15) and (16), respectively.

$$
\begin{gathered}
\varphi_{f}=\left\{\varphi_{1 F}, \varphi_{2 F}, \varphi_{3 F}, \varphi_{4 F}\right\} \\
f_{M f}=\left\{f_{M F 1}, f_{M F 2}, f_{M F 3}, f_{M F 4}\right\}
\end{gathered}
$$

Similarly the local phase and local amplitude values are calculated for the IMF's and residue of iris and fingerprint. Hence $\varphi_{i}$ and $f_{M i}$ for iris and $\varphi_{f p}$ and $f_{M f p}$ for fingerprint.

Grey level co-occurrence matrix: The images which is enhanced by the ideal high pass filters are given as an input to the GLCM. It is used for extracting the second order statistical texture features and it is introduced by Haralick. GLCM transforms the image into a matrix with respect to the pixels of an original image. The calculation of mutual occurrence of pixel pairs is required and it is calculated for a specific distance oriented at a particular direction. Then the statistical features are extracted for further process. There are different types of features are extracted from the GLCM of pre-processed face image and the expression of features are given in below Eq. (17)-(27).

Auto correlation

$$
F 1=\sum_{m} \sum_{n}(m n) X_{F}(m, n)
$$


Contrast

$$
F 2=\sum_{i=0}^{N_{g}-1} i^{2}\left\{\sum_{i=1}^{N_{g}} \sum_{j=1}^{N_{g}} X_{F}(m, n)\right\}
$$

Correlation

$$
F 3=\frac{\sum_{i} \sum_{j}(m n) X_{F}(m, n)-\mu_{x} \mu_{y}}{\sigma_{x} \sigma_{y}}
$$

Energy

$$
F 4=\sum_{i} \sum_{j} X_{F}(m, n)^{2}
$$

Entropy

$$
F 5=\sum_{i} \sum_{j} X_{F}(m, n) \log \left(X_{F}(m, n)\right)
$$

Homogeneity

$$
F 6=\sum_{i} \sum_{j} \frac{1}{1+(m-j)^{2}} X_{F}(m, n)
$$

Sum average

$$
F 7=\sum_{m=2}^{2 N_{g}} m X_{F x+y}(m)
$$

Sum entropy

$$
F 8=-\sum_{i=2}^{2 N_{g}} X_{F x+y}(m) \log \left(p_{x+y}(m)\right)
$$

Sum variance

$$
F 9=\sum_{m=2}^{2 N_{g}}(m-F 8)^{2} X_{F x+y}(m)
$$

Difference variance

$$
F 10=\text { varianceof } X_{F x-y}
$$

Difference entropy

$$
F 11=-\sum_{m=0}^{N_{g-1}} X_{F x-y}(m) \log \left(X_{F x-y}(m)\right)
$$

where, $X_{F}(m, n)$ is the entry of a normalized GLCM, $N_{g}$ is the number of distinct grey levels in the quantized image [22].
4) Feature Level Fusion (FLP) : FLF receives the feature vectors from the hybrid feature extraction technique and it has the rich information about the biometric features. This FLF performs before matching and performs two levels of fusion. At first, this FLF fuses the features from the HHT with GLCM for face, iris, and fingerprint individually. In second level fusion, the fusion vectors of face, iris, and fingerprint are fused together.

The response time of the feature level fusion is less than the score level fusion. FLF concatenates the extracted features. The dimensionality of the fused feature vector maximizes by the feature set of concatenation. The steps which are performed in the FLF are:

- Normalization of feature vector.

- Fusing the feature vector.

Normalization of feature vector: The feature vectors which are extracted from face, iris and fingerprint are incompatible in nature. Because of the variation in its own range and distribution. This problem overcome by normalizing the feature vector.

Fusing the feature vector: The final fused vector is achieved by concatenating the feature vector from face, iris and fingerprint.The expression for first level fusion is given in Eq. (28), (29) and (30).

$$
\begin{gathered}
f=\left[\varphi_{f}, f_{M f}, F\right] \\
i=\left[\varphi_{i}, f_{M i}, I\right] \\
f p=\left[\varphi_{f p}, f_{M f p}, F P\right]
\end{gathered}
$$

Where $f, i$ and $f p$ are first level fusion vectors of face, iris and fingerprint respectively.In second level fusion, these features are combined together and the fused vector $\left(z_{v}\right)$ is shown in the following Eq. (31).

$$
\mathrm{z}_{v}=\left[f_{1}, f_{2}, \ldots f_{n} i_{1}, i_{2}, \ldots i_{n} f p_{1}, f p_{2}, \ldots f p_{n}\right]
$$

Where, $f_{1}, f_{2}, \ldots f_{n}, i_{1}, i_{2}, \ldots i_{n}$ and $f p_{1}, f p_{2}, \ldots f p_{n}$ defines the normalized vectors of face, iris and finger print, respectively. These fused vectors are stored in the database and it is used for the identification of individuals.

Furthermore, the extracted features of face, iris and fingerprint are stored into the database. Then it is transferred to the MCSVM for testing.

\section{B. MMB-BEMD-HHT Training by MC-SVM}

The fused vector data about the face, iris and fingerprint are trained in the MC-SVM classifier. MC-SVM is a pattern classification algorithm which depends on the statistical learning theory [23]. A small classification samples (nonlinear samples) and high dimensional problems overcome by using SVM and it depends on the Structural Risk Minimization (SRM) which reduces the upper bound over the expected risk in contrast. The basic principle behind the SVM is to resolve the classification problems. The basic structure for SVM is shown in Fig. 3. 


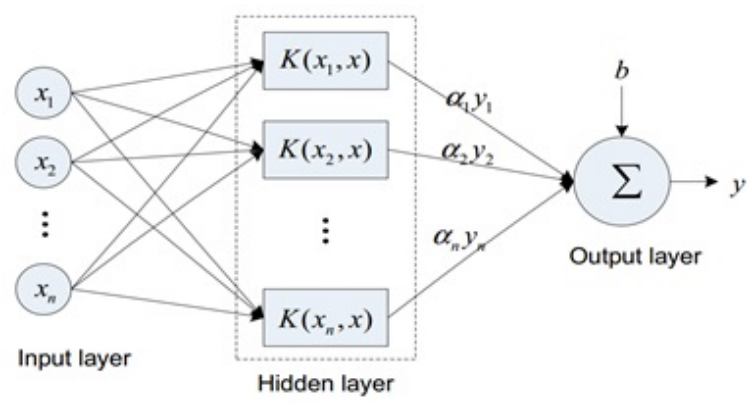

Fig. 3. Structure of SVM

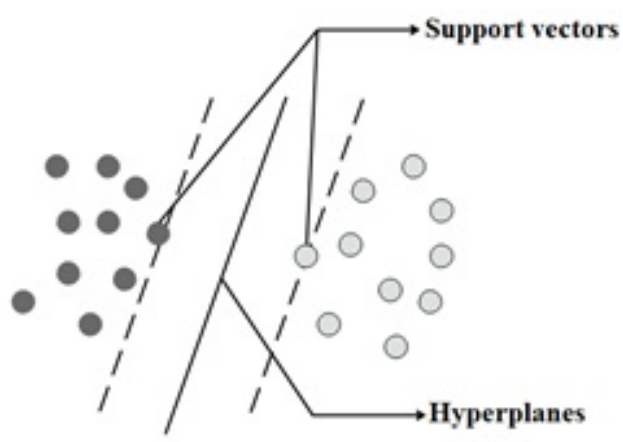

Fig. 4. Linearly separable data with multiple hyper plane.

The SVM is a two class SVM that separate the data into two classes by identifying the hyper plane among the data with maximum margin. There may be multiple numbers of hyper planes present inside the SVM. MMB-BEMD-HHT has to find the optimal hyper plane that separates the data in error free and also increases the margin among the two classes. Fig. 4 and 5 shows the linear separable data with multiple and optimum hyper plane, respectively. Then the nonlinear separable data is shown in Fig. 6.

SVM has a kernel function which performs the separating function and this function use for determining the optimal hyper plane. The kernel function of SVM is an integral part of SVM that divides the input data into two classes involving a less number of support vectors. There are four types of kernel functions that is given below.

1) Linear, $K\left(z_{i}, z_{j}\right)=z_{i}^{T} \mathrm{z}$

2) Polynomial, $K\left(z_{i}, z_{j}\right)=\left(\gamma z_{i}^{T} z_{j}+r\right)^{d}, \gamma>0$

3) Radial Basis Function (RBF),

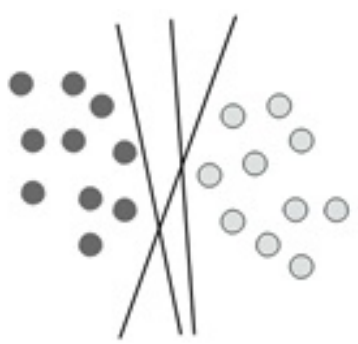

Fig. 5. Linearly separable data with optimum hyper plane.

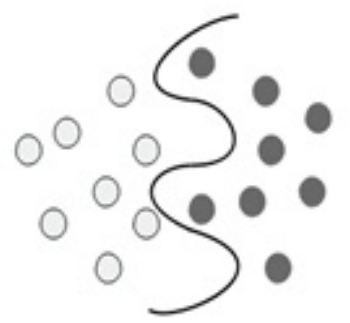

Fig. 6. Nonlinear separable data.

$$
\begin{aligned}
& K\left(z_{i}, z_{j}\right)=\exp \left(-\gamma\left\|z_{i}-z_{j}\right\|^{2}\right), \gamma>0 \\
& \text { 4) } \quad \text { Sigmoid, } K\left(z_{i}, z_{j}\right)=\tanh \left(z_{i}^{T} z_{j}+r\right)
\end{aligned}
$$

Where, $r, \gamma$ and $d$ are the kernel parameters. The kernel function is chosen based on the classification problem. From the four kernel functions, the radial basis function (RBF) is chosen because the samples are non-linearly mapped to a high dimensional space. The relation among class labels and the non-linear attributes of class labels are managed by the RBF.

1) Multi Class Classification of SVM : SVM is employed for binary class problem. Then the SVM is extended to a multi class SVM. The conversion of SVM to Multi class SVM has two strategies such as one against one strategy and one against rest strategy. One against one strategy use to classify between each pairs and one against rest strategy classifies among each class and all the remaining biometric traits. These two schemes use to create the multiclass SVM. The one against one strategy needs a $k(k-1) / 2$ binary SVMs and one against rest strategy needs a $k$ binary SVMs to build a $k$ classifier.Finally, the trained values from the MC-SVM is stored as a database and it is given to the input for testing process.

2) $M M B-B E M D-H H T$ Testing by MC-SVM: Image Acquisition is the process of capturing the biological input image. Then the captured input image is enhanced by the high-pass filter. The pre-processed image feature values are extracted by the HHT and GLCM which is similar to feature extraction. Then the feature values are fused by the FLF fusion method that is given in feature level fusion. The fused feature values are given to MC-SVM prediction with the trained features of face, fingerprint and iris that predicts whether the tested image is authenticated or not.This MC-SVM is used for classifying an individual biometric trait.

\section{RESUlTS AND Discussion}

The MMB-BEMD-HHT method was analysed with the help of MATLAB 2017b. This MMB-BEMD-HHT method was developed with the biometric features of face, iris and fingerprint to enhance the security of the desired system. Here, the database for face, iris and fingerprint are MIT CBCL, MMU and Casia V5 respectively [26], [27], [28]. The performance of the MMB-BEMD-HHT was evaluated in terms of FAR, FRR and accuracy.

In MMB-BEMD-HHT method, the particular person is identified by anyone of the biometric feature like face, iris and fingerprint. Total 150 images were used in MMB-BEMD-HHT training and this 150 images comprised of 50 face images, 50 

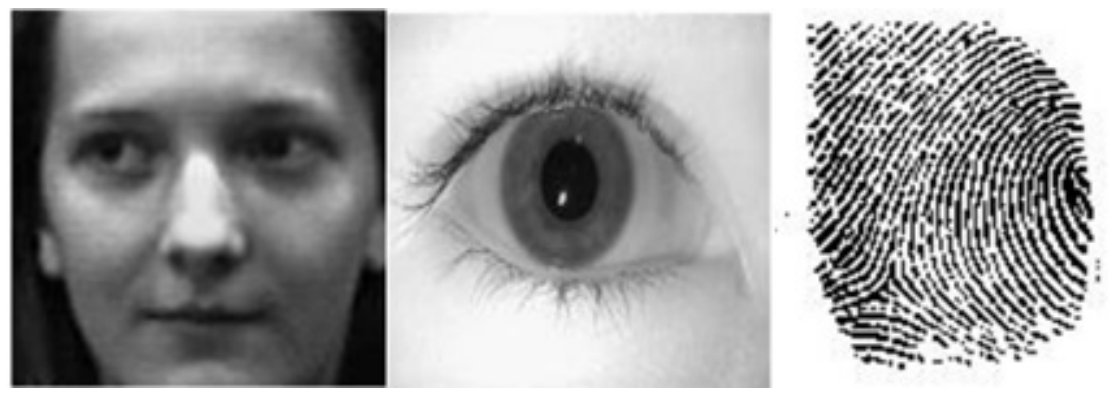

Fig. 7. Pre-processed images of face, iris and fingerprint.
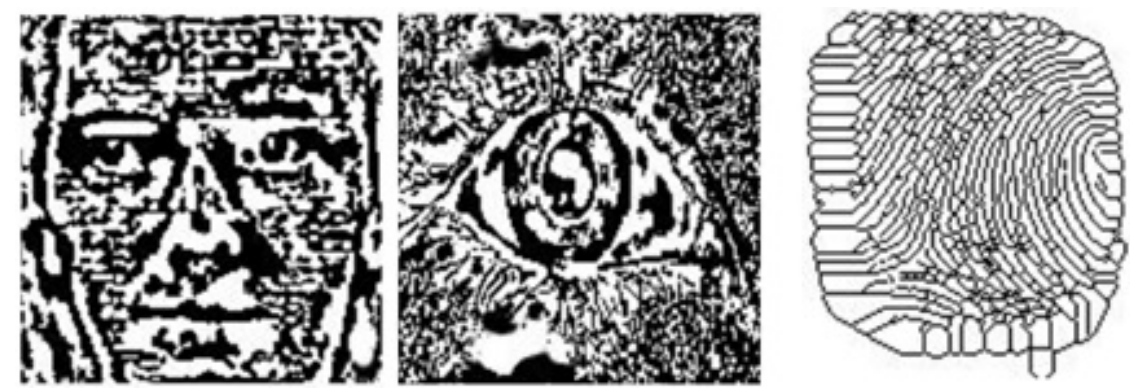

Fig. 8. Feature extracted images of face, iris and fingerprint.

TABLE I. COMPARATIVE ANALYSIS OF MMB-BEMD-HHT

\begin{tabular}{llll}
\hline \hline Parameters & SIFT-KNN-MM-BS [24] & FLF-FFF-MM-BS [25] & MMB-BEMD-HHT \\
\hline FAR & $3.75 \%$ & $5 \%$ & $0.10 \%$ \\
FRR & $7.5 \%$ & $5 \%$ & $2.0 \%$ \\
Accuracy & $92.5 \%$ & $95 \%$ & $96 \%$ \\
\hline \hline
\end{tabular}

\begin{tabular}{|c|c|c|}
\hline $\begin{array}{c}1^{\text {st }} \text { Face image BEMD } \\
\text { feature values }\end{array}$ & $\begin{array}{c}1^{\text {st }} \text { Iris image BEMD } \\
\text { feature values }\end{array}$ & $\begin{array}{c}1^{\text {st }} \text { Fingerprint image } \\
\text { minutiae } \\
\text { feature values }\end{array}$ \\
\hline
\end{tabular}

Fig. 9. Example of fusion structure.

\begin{tabular}{|c|}
\hline $1^{\text {st }}$ face, $1^{\text {st }}$ iris $\& 1^{\text {st }}$ fingerprint database value \\
\hline $2^{\text {nd }}$ face, $2^{\text {nd }}$ iris $\& 2^{\text {nd }}$ fingerprint database value \\
\hline $3^{\text {rd }}$ face, $3^{\text {rd }}$ iris $\& 3^{\text {rd }}$ fingerprint database value \\
\hline \\
\hline $50^{\text {th }}$ face, $50^{\text {th }}$ iris $\& 50^{\text {th }}$ fingerprint database value \\
\hline
\end{tabular}

Fig. 10. Database structure for MMB-BEMD-HHT.

iris images and 50 fingerprint images of 10 persons. Here, the biometric traits of single person have 5 face images, 5 iris images and 5 fingerprint images. Initially, the pre-processing process takes place to enhance the clarity of biometric traits. The pre-processed images of face, iris and fingerprint are shown in Fig. 7. Then the features of pre-processed images were extracted by HHT and GLCM.The feature extracted images are shown in Fig. 8. After extracting the features, it was stored in the database. Later, these features were tested in MC-SVM for testing. There are 60 images are used in the testing of MMB-BEMD-HHT and these 60 images comprises of 20 face images, 20 iris images and 20 fingerprint images of 10 persons. The pre-processing and feature extraction of MMB-BEMD-HHT testing are similar to the training. Finally, the features of the MMB-BEMD-HHT testing were compared with the trained features of face, iris and fingerprint to identify the features of individuals.

The extracted BEMD features of face, iris and fingerprint were fused by FLF and the example of FLF structure along with face, iris and fingerprint features are shown in Fig. 9. Then these feature values were stored in the database and the database structure is shown in Fig. 10.

The input images used in the MMB-BEMD-HHT training are taken as an input to the testing section. Testing also takes three different biometrics such as face, iris and fingerprint. HHT and GLCM and FLF fusion of testing are similar to the MMB-BEMD-HHT training. Based on the MC-SVM, the similarity between the database images to specific person is identified.

Fig. 11 shows the example of the class allocation for different images in the database. The first and second row represents the pictures and the respective classes of the images. 


\begin{tabular}{|c|c|c|c|c|c|c|c|c|c|c|c|c|c|}
\hline Pictures & P1 & $\mathrm{P} 2$ & P3 & P4 & P5 & P6 & P7 & P8 & \begin{tabular}{l|l} 
P9 & P10
\end{tabular} & -ーーーー & \begin{tabular}{|l|l|} 
P46 & P47 \\
\end{tabular} & \begin{tabular}{|l|l|} 
P48 & P49 \\
\end{tabular} & P50 \\
\hline Class & & & $\mathrm{Cl}$ & & & & & $\mathrm{C} 2$ & & י-ーーーー & & $\mathrm{C} 50$ & \\
\hline
\end{tabular}

Fig. 11. Class allocating for MMB-BEMD-HHT.

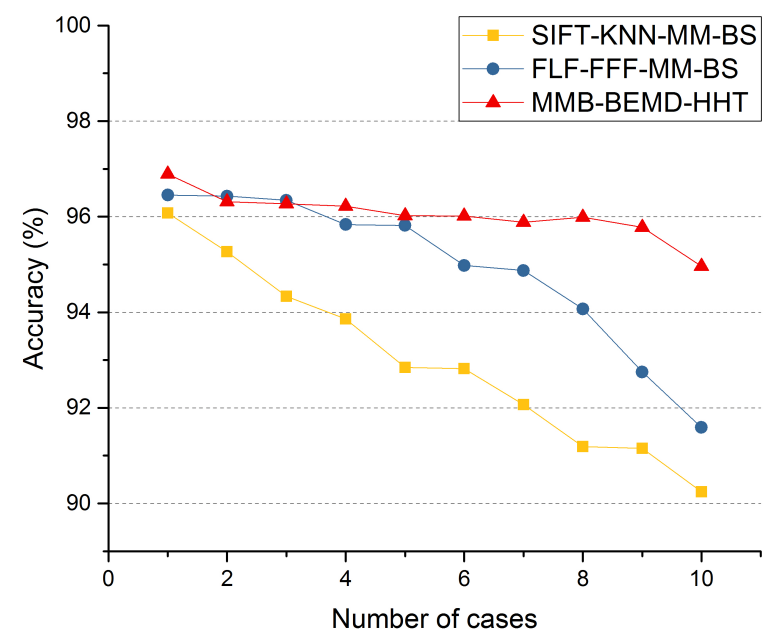

(a)

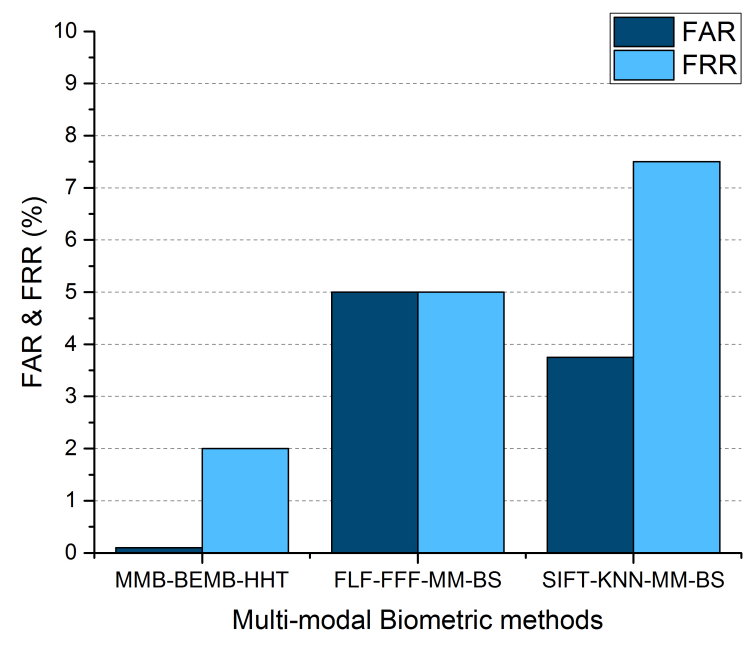

(b)

Fig. 12. Comparison of MMB-BEMD-HHT Vs existing methods (a) Accuracy (b) FAR and FRR.

From the classes, the correlated values are discovered to find the true positive (TP), true negative (TN), false positive (FP) and false negative (FN). A true positive is an outcome where the model correctly predicts the positive class. Similarly, a true negative is an outcome where the model correctly predicts the negative class. A false positive is an outcome where the model incorrectly predicts the positive class. And a false negative is an outcome where the model incorrectly predicts the negative class. These values helps to determine the following performance measures like False acceptance ratio (FAR), False Rejection Ratio (FRR) and accuracy using Eq. (32)-(34).

1) False acceptance ratio (FAR): FAR is the measure of the Biometric Security System (BSS) which incorrectly accepts an unauthorized operator. The following Eq. (32) describes the FAR mean.

$$
F A R=\frac{F P}{F P+T N}
$$

2) False Rejection Ratio (FRR): FRR is the measure of the likelihood which incorrectly reject an access attempt by an authorized user in MM-BS. The expression for FRR is given in the following Eq. (33).

$$
F R R=\frac{F P}{T P+F N}
$$

3) Accuracy (A): The accuracy of the respective image determine based on the specificity $(S \mid p)$ and $\operatorname{sensitivity}\left(S_{e}\right)$. Quantity of the image is accurately represented by using the following Eq. (34).

$$
A=\frac{T P+T N}{T P+F P+T N+F N}
$$

The MMB-BEMD-HHT is compared with two existing methods, which is explained below.

In first method,the image description and feature extraction of face and fingerprint images were performed by Scale Invariant Feature Transform (SIFT) and then the classification of biometric traits were made by $\mathrm{K}$ Nearest Neighbour (KNN) classifier. A SIFT algorithm used in this MM-BS was based on the gradient of each pixel. The pixel values which were affected by the noise is eliminated by cascade filtering technique. The removal of noise pixels affects the recognition performance [24]. In second method, the finger knuckle and finger vein were taken in MM-BS. A repeated line tracking method was used to extract the features from the finger knuckle and finger vein. Then these image features are fused by FLF with fractional firefly (FFF) optimization. Here, the layered $\mathrm{k}-\mathrm{SVM}$ to carry out the recognition [25].

Table I shows the performance analysis of MMB-BEMDHHT technique. Fig. 12 shows the comparative analysis of the MMB-BEMD-HHT Vs existing methods. Here the accuracy is compared with existing methods, because in real time systems accuracy plays a crucial role in image recognition. From the comparison, conclude that the MMB-BEMD-HHT method gives better performance in terms of accuracy as well the other parameters like FAR, and FRR also improved. By improving the accuracy of the desired system, the authentication of an individual person becomes easy. 


\section{CONCLUSION}

MMB-BEMD-HHT method is developed based on the three different biometric traits of face, iris and fingerprint to improve the accuracy in finding the individuals. In MMBBEMD-HHT method, the images of face, iris and fingerprints are enhanced by using the high pass filter. The features of the image is extracted by HHT and GLCM. Then these feature vectors are fused by FLF. MC-SVM is used as the classifier and it verifies the person's identity based on the feature vectors which is stored in the database. The performance of MMBBEMD-HHT is analysed with an existing technique. The experimental results show that the MMB-BEMD-HHT gives $96 \%$ of accuracy, $0.10 \%$ of FAR and $2 \%$ of FRR for multimodal biometric recognition. The MMB-BEMD-HHT gives better accuracy of $96 \%$ compared to the existing methods. Furthermore, the optimization technique can be used in the feature extraction technique which may improve recognition accuracy. The proposed method is worked on limited dataset, in next phase more datasets with attributes will be considered.

\section{COMPLIANCE WITH ETHICAL STANDARDS}

Conflict of Interests: Conflict of Interests: The authors of this paper declare that there is no conflict of interests regarding the publication of this manuscript.

Ethical Approval: This article does not contain any studies with human participants or animals performed by any of the authors.

\section{ACKNOWLEDGMENTS}

The authors acknowledge the Massachusetts Institute of Technology, Multimedia University, Malaysia and Chinese Academy of Sciences for critical role in the creation of publicly available database of face, iris and fingerprint respectively, used in this study.

\section{REFERENCES}

[1] D. J. Ghate and S. B. Patil, "Survey of Biometric , Multimodal Biometric Systems," International Journal of Science and Research (IJSR), vol. 3, no. 11, pp. 2012-2015, 2014.

[2] A. T. Siddiqui, "An Enhanced Multi-Modal Biometric System for Secure User Identification," Asian Journal of Technology and Management Research, vol. 06, no. 01, pp. 2249-892, 2016.

[3] K. S. Wu, J. C. Lee, T. M. Lo, K. C. Chang, and C. P. Chang, "A secure palm vein recognition system," Journal of Systems and Software, vol. 86, no. 11, pp. 2870-2876, 2013. [Online]. Available: http://dx.doi.org/10.1016/j.jss.2013.06.065

[4] W. Yang, S. Wang, J. Hu, G. Zheng, and C. Valli, "A fingerprint and finger-vein based cancelable multi-biometric system," Pattern Recognition, vol. 78, pp. 242-251, 2018. [Online]. Available: https://doi.org/10.1016/j.patcog.2018.01.026

[5] M. V. Karki and S. S. Selvi, "Multimodal Biometrics at Feature Level Fusion using Texture Features," International Journal of Biometrics and Bioinformatics (IJBB), vol. 7, no. 1, pp. 58-73, 2013.

[6] D. Jagadiswary and D. Saraswady, "Biometric Authentication Using Fused Multimodal Biometric," in Procedia Computer Science, vol. 85, no. Cms. Elsevier Masson SAS, 2016, pp. 109-116. [Online]. Available: http://dx.doi.org/10.1016/j.procs.2016.05.187

[7] G. Amirthalingam and G. Radhamani, "New chaff point based fuzzy vault for multimodal biometric cryptosystem using particle swarm optimization," Journal of King Saud University - Computer and Information Sciences, vol. 28, no. 4, pp. 381-394, 2016. [Online]. Available: http://dx.doi.org/10.1016/j.jksuci.2014.12.011
[8] F. S. Assaad and G. Serpen, "Transformation based Score Fusion Algorithm for Multi-modal Biometric User Authentication through Ensemble Classification," in Procedia Computer Science, vol. 61. Elsevier Masson SAS, 2015, pp. 410-415. [Online]. Available: http://dx.doi.org/10.1016/j.procs.2015.09.175

[9] F. Alsaade, "Neuro-fuzzy logic decision in a multimodal biometrics fusion system," Scientific Journal of King Faisal University, vol. 11, no. 2, pp. 163-177, 2010.

[10] Y. G. Kim, K. Y. Shin, E. C. Lee, and K. R. Park, "Multimodal biometric system based on the recognition of face and both irises," International Journal of Advanced Robotic Systems, vol. 9, pp. 1-6, 2012.

[11] M. S. Panag and T.S., "Heterogeneous Multimodal Biometric System with Fuzzy Vault Template Security," International Journal of Advanced Research in Computer Science and Software Engineering, vol. 4, no. 9, 2014.

[12] O. M. Aly and T. A. Mahmoud, "An Adaptive Multimodal Biometrics System using PSO," International Journal of Advanced Computer Science and Applications, vol. 4, no. 7, pp. 158-165, 2013.

[13] J. Peng, A. A. El-Latif, Q. Li, and X. Niu, "Multimodal biometric authentication based on score level fusion of finger biometrics," Optik, vol. 125, no. 23, pp. 6891-6897, 2014. [Online]. Available: http://dx.doi.org/10.1016/j.ijleo.2014.07.027

[14] G. K. O. Michael, T. Connie, and A. B. J. Teoh, "A contactless biometric system using multiple hand features," Journal of Visual Communication and Image Representation, vol. 23, no. 7, pp. 1068-1084, 2012. [Online]. Available: http://dx.doi.org/10.1016/j.jvcir.2012.07.004

[15] S. T. Krishna Shinde, "Development of Face and Signature Fusion Technology for Biometrics Authentication," International Journal of Emerging Research in Management \&Technology, vol. 9359, no. 9, pp. 61-65, 2017.

[16] W. Yang, S. Wang, G. Zheng, and C. Valli, "Impact of feature proportion on matching performance of multi-biometric systems," in ICT Express. Elsevier B.V., 2018, pp. 1-5. [Online]. Available: https://doi.org/10.1016/j.icte.2018.03.001

[17] P. Gupta and P. Gupta, "Multi-modal fusion of palm-dorsa vein pattern for accurate personal authentication," Knowledge-Based Systems, vol. 81, no. March, pp. 117-130, 2015. [Online]. Available: http://dx.doi.org/10.1016/j.knosys.2015.03.007

[18] M. Xin and J. Xiaojun, "Correlation-based identification approach for multimodal biometric fusion," Journal of China Universities of Posts and Telecommunications, vol. 24, no. 4, pp. 34-39-50, 2017. [Online]. Available: http://dx.doi.org/10.1016/S1005-8885(17)60221-8

[19] M. S. Mohd Asaari, S. A. Suandi, and B. A. Rosdi, "Fusion of Band Limited Phase only Correlation and Width Centroid Contour Distance for finger based biometrics," Expert Systems with Applications, vol. 41, no. 7, pp. 3367-3382, 2014. [Online]. Available: http://dx.doi.org/10.1016/j.eswa.2013.11.033

[20] C. M. Patil and S. Patilkulkarni, "An efficient process of recognition of human iris based on contourlet transforms," Procedia Computer Science, vol. 2, no. 2009, pp. 121-126, 2010. [Online]. Available: http://dx.doi.org/10.1016/j.procs.2010.11.015

[21] C. M. Patil, "An Efficient Iris Recognition System to Enhance Security Environment for Personal Identification," International Journal of Communications Networking System, vol. 2, no. 12, pp. 174-179, 2013.

[22] L. K. Soh and C. Tsatsoulis, "Texture analysis of sar sea ice imagery using gray level co-occurrence matrices," IEEE Transactions on Geoscience and Remote Sensing, vol. 37, no. 2 I, pp. 780-795, 1999.

[23] P. Sharma and M. Kaur, "Multimodal Classification using Feature Level Fusion and SVM," International Journal of Computer Applications, vol. 76, no. 4, pp. 26-32, 2013.

[24] G. W. Mwaura, P. W. Mwangi, and C. Otieno, "Multimodal Biometric System :- Fusion Of Face And Fingerprint Biometrics At Match Score Fusion Level," International Journal of Scientific and Technology Research Volume, vol. 6, no. 04, pp. 41-49, 2017.

[25] S. Veluchamy and L. Karlmarx, "System for multimodal biometric recognition based on finger knuckle and finger vein using feature-level fusion and k-support vector machine classifier," IET Biometrics, vol. 6, no. 3, pp. 232-242, 2017. [Online]. Available: http://digitallibrary.theiet.org/content/journals/10.1049/iet-bmt.2016.0112 
[26] B. Weyrauch, B. Heisele, J. Huang, and V. Blanz, "Component-based face recognition with 3D morphable models," in IEEE Computer Society Conference on Computer Vision and Pattern Recognition Workshops, vol. 2004-Jan, no. January, 2004.
[27] Malaysia Multimedia University. MMU1 iris image database, 2004. [Online]. Available: http://pesona.mmu.edu.my/ ccteo/

[28] "Casia iris image database." Institute of Automation, Chinese Academy of Sciences, 2012. [Online]. Available: http://biometrics.idealtest.org/ 